\title{
Investigation of Yield Regulation of Red Grapes on the Concentration of Some Crucial Wine Components
}

\author{
Kinga K. Rácz ${ }^{1 *}$, Miklós Kállay¹, Nóra Bakos-Barczi², László Rácz³ , Csaba Csutorás³ \\ ${ }^{1}$ Department of Viniculture, Corvinus University, Budapest, Hungary \\ ${ }^{2}$ Eger Crown Winehouse Ltd., Demjén, Hungary \\ ${ }^{3}$ Institute of Food Science, Eszterházy Károly University, Eger, Hungary \\ Email: *racz.kinga@koronaborhaz.hu
}

Received 7 January 2016; accepted 24 April 2016; published 29 April 2016

Copyright (C) 2016 by authors and Scientific Research Publishing Inc.

This work is licensed under the Creative Commons Attribution International License (CC BY).

http://creativecommons.org/licenses/by/4.0/

(c) () D Open Access

\begin{abstract}
An essential concept of making quality wine is that the quality comes from the vineyard. The dissolved materials and components determine the value of wine. Their amount and proportion are essentially influenced by grape variety, terroir, the way of cultivation, weather conditions, grape quality, the time of vintage and the method of grape and wine processing. In the present study the effect on wine quality of different ways of yield regulations was examined in wines of three consecutive years. Besides basic parameters, total polyphenol and resveratrol contents were determined in wines of Blaufränkisch and Merlot varieties. Our measurements were carried out by spectrophotometry, gas and liquid chromatography. Our investigations confirmed that yield regulation led to increased extract content and outstanding quality of the produced wine.
\end{abstract}

\section{Keywords}

Yield Regulation, Polyphenol, Resveratrol, Gas Chromatography, Liquid Chromatography

\section{Introduction}

The adjustment of the optimal crop load in order to achieve expected wine quality is still the most discussed viticultural topic. One of the best known and most widely used routines within the methods of yield regulation is cluster thinning. However, there are several other alternative ways to lower the yield in the vineyard, like cluster tipping, defoliation at flowering and cluster shredding. Incision on vines combined with sprout selection is

${ }^{*}$ Corresponding author.

How to cite this paper: Rácz, K.K., Kállay, M., Bakos-Barczi, N., Rácz, L. and Csutorás, C. (2016) Investigation of Yield Regulation of Red Grapes on the Concentration of Some Crucial Wine Components. Agricultural Sciences, 7, $279-286$.

http://dx.doi.org/10.4236/as.2016.74027 
another special method of yield regulation leading to better quality of grape and wine. It is also widely believed by wine growers that high-yielding vines produce lower-quality wines. This thought should be considered carefully as it depends primarily on fertility and quality of the cultivar and on growing conditions of the vineyard [1]. The relationship between crop level and wine quality was widely investigated and reviewed. Two major measures are employed for evaluating the crop load: leaf area to yield ratio and yield to pruning weight ratio, which is more practical for field measurements [2] [3]. The influence of different crop loads on grape and wine quality for different cultivars and different wine growing regions has been extensively described and reviewed in the last decades [4]-[6]. Applying cluster tipping or shredding, for example, results in looser cluster structure, thus the risk of gray rot or vinegar production can be lowered. It improves not only the crucial chemical components of must and wine, but condition of grapevines can be also optimized. Many authors mention that especially in the case of red grapes higher extract and anthocyanin content can be obtained as a result of yield regulation.

Flavonoids are the most abundant polyphenols that contain two or more aromatic rings connected to at least one aromatic hydroxyl group and a bridge of carbon. Recently these compounds have been the subject of many studies since these are identified with health benefits, ranging from prevention of caries to cancer. Much has been said about the functionality of these compounds that have anti-carcinogenic, anti-atherogenic, anti-thrombotic, anti-microbial, and analgesic vessel dilator effects [7] [8]. Some studies regarding the functionality of these compounds relate to their presence in wines which possess large amounts of flavanols, antioxidant substances that fight free radicals [9]. Although flavonoids are colorless or slightly yellowish, they have an important role in strengthening and stabilizing the color of red wines. They contribute to the sensory characteristics such as taste, astringency and harshness of wine, in addition to the preservation of beverages and retaining properties of wine aging process. Most phenols are in the form of tannins (molecules which preserve wine by absorbing oxygen) or as flavonoid derivatives such as flavan-3-ols and flavan-3,4-diols that can polymerize tannins [10] [11].

Spectrophotometric and chromatographic methods were developed for the analysis of different polyphenols. GC and HPLC techniques can be used for the determination of a wide range of wine polyphenols as anthocyanins, leucoanthocyanin, catechin, resveratrol and piceid [12]-[14]. Validated HPLC methods were developed for the quantitative analysis of trans-resveratrol and trans-piceid that were recently in the focus of many researches [15] [16]. Blaufränkisch and Cabernet Franc wines were recently studied, authors found correlation between chemical and sensory characteristics. Total polyphenolic content, anthocyanin, leucoanthocyanin and catechin were evaluated by spectrophotometry stilbenes and were identified and quantified by HPLC [17].

In our paper we focus on the investigation of the effect of yield regulation in the vineyard on the quality parameters of red wines. In our study we intended to find correlation between organoleptic characteristics and phenolic compound content by using yield regulation of different extent.

\section{Materials and Methods}

\subsection{Yield Regulation Experiments}

The experiments were carried out in two leading terroirs of Eger Crown Winehouse Ltd., in Eger wine region. The vineyard of the experiment was planted in 2003 in brown forest soil formed on volcanic rhyolite tuff. The plantation is located in South-West orientation the rows are in North-South direction. The examined grape varieties were Blaufränkisch and Merlot. The applied cultivation method was umbrella cultivation combined with arced, cane fixing method. The trunk height is $80-90 \mathrm{~cm}$. The row-and vine distance in the plantation is $240 \times$ $100 \mathrm{~cm}$. Yield regulation experiments were carried out in three following years (2011-2012-2013), at 100\%, 80\% and $60 \%$ load levels. In the experiment control vines without yield regulation (100\%) were compared with vines of different load of $80 \%$ and $60 \%$ that was achieved by cluster selection. In the case of the control $(100 \%)$ there was no cluster removal but at the $80 \%$ load level there was $20 \%$ yield regulation accomplished. At the $60 \%$ load level the yield regulation was $40 \%$ namely $40 \%$ of all the clusters was removed at sprouting, at the end of July, beginning of August.

Both Blaufränkisch and Merlot grapes were harvested in full ripeness with $20^{\circ}$ and $21^{\circ}$ sugar degree of the must. Wine was made from each grape by identical microvinification methods, therefore differences could be only resulted by yield regulation. The grape was treated with sulfur $(15 \mathrm{~g} / \mathrm{hL})$, then after destemming yeast (doses of $30 \mathrm{~g} / \mathrm{hL}$ ) was added to start fermentation in $50 \mathrm{~L}$ covered plastic barrels. Fermentation was carried out at cellar temperature $\left(12^{\circ} \mathrm{C}-13^{\circ} \mathrm{C}\right)$, sugar decrease was regularly checked. In case of all wines the skin macera- 
tion time was 20 days. After complete fermentation sulfurization was done to set free sulfurous acid level to 30 $\mathrm{mg} / \mathrm{L}$, then the barrels were sealed and after self-clarification wines were bottled for analyses.

\subsection{Spectrophotometric Determinations of Polyphenols}

Absorbance measurements were recorded on a Beckmann DU 530 spectrophotometer. Total polyphenolic content in wine samples was determined by the Folin-Ciocalteu's reagent [18] and the results were expressed in $\mathrm{mg} / \mathrm{L}$ of gallic acid equivalent. Anthocyanins were estimated according to Ribéreau and Stonestreet [19] and were expressed in $\mathrm{mg} / \mathrm{L}$. The content of leucoantocyanins was measured after heating wine with 40:60 hydrochloric acid and butanol, containing $300 \mathrm{mg} / \mathrm{L}$ ferro-sulphate [20]. Catechin was determined by using the REBELEIN method [21], after diluting wine with alcohol and reacting with vanillin sulfuric acid. Folin-Ciocalteu's reagent of analytic purity, sodium carbonate, 99\% gallic acid (3,4,5-trihidroxi-benzoic acid) and all the reagents were purchased from Sigma-Aldrich Ltd. (Budapest, Hungary).

\subsection{High-Performance Liquid Chromatography Analysis}

Concentrations of stilbenes were determined by using high-performance liquid chromatography (HPLC) method with isocratic elution with a Thermo Scientific Dionex UltiMate 3000 Instrument equipped diode array UVvisible detector. The isocratic solvent consisted of $90 \%$ water, $5 \%$ methanol and $5 \%$ acetonitrile. All wines were analyzed in terms of four stilbenes: trans-resveratrol, cis-resveratrol, trans-piceid and cis-piceid [15]. The wine samples were analyzed after filtration by Minisart Syringe Filter (Minisart RC Hydrophilic, $15 \mathrm{~mm}, 0.2 \mu \mathrm{m}$ ) and kept from light at $4^{\circ} \mathrm{C}$. The circumstances of the HPLC-measurement were as follows: The system was equipped with a Hypersil GOLD column C18 $(250 \times 4 \mathrm{~mm}, 3 \mu \mathrm{m}$ particle size $)$ with a pre-column $(10 \times 4 \mathrm{~mm}, 3 \mu \mathrm{m}$ particle size) of the same material, maintained at $35^{\circ} \mathrm{C}$. Mobile phase consisted of a water/methanol/acetic acid mixture (volume ratio 64.6/33.4/2) with a flow rate of $1.0 \mathrm{~mL} / \mathrm{min}$. Injection volume was $20 \mu \mathrm{L}$. Detection was performed at $310 \mathrm{~nm}$ wavelength, run time was $15 \mathrm{~min}$. Quantification was based on peak area. The content of total resveratrol was expressed in $\mathrm{mg} / \mathrm{L}$ of wines. Analysis was based on calibration with commercially available standards (Sigma Aldrich, St. Louis, MO, USA). Detection limit was $0.1 \mathrm{mg} / \mathrm{L}$ for all components.

Trans-Resveratrol and Piceid standards of analytical purity were purchased from Sigma-Aldrich Ltd. (Budapest, Hungary). Both the standards of resveratrol and piceid were trans-isomers, cis-isomers were synthesized by treating the trans-isomers for 30 minutes by UV irradiation. Methanol, HPLC solvents, acetic acid were purchased from VWR (Debrecen, Hungary).

\subsection{Organoleptic Analyses}

The sensory examination and evaluation of wines was carried out by wine tasting. To ensure impartial judgment of wine, they were served blind for 8 experts, who set up the order in the case of each different wine. The best one from each variety was analyzed according to the commonly used 100 scale wine tasting method and the results were displayed with the help of radar chart.

\subsection{Data Analyses}

The statistical evaluation was carried out by the commonly used Student's t-test. A two-sample location test of the null hypothesis of two different yield regulations has an equal result in the aspect of total polyphenol content, color intensity or color tone.

\section{Results and Discussion}

\subsection{Total Polyphenol Content}

The measured total polyphenol values are shown in Figure 1 and Figure 2.

In the case of Blaufränkisch and Merlot wines similar values for total polyphenol content can be seen.

Both figures show that the year of vintage definitely determines the polyphenol content of wine. Year 2012 was weaker, than the 2011 and 2013 vintages. The total polyphenol content of 2012 wines was lower even at $60 \%$ yield regulations, than without yield regulation in the other two years (2011 and 2013). 80\% and 60\% yield regulations were proved to be effective in all three years, in the case of both red wines. Regarding the increase of 


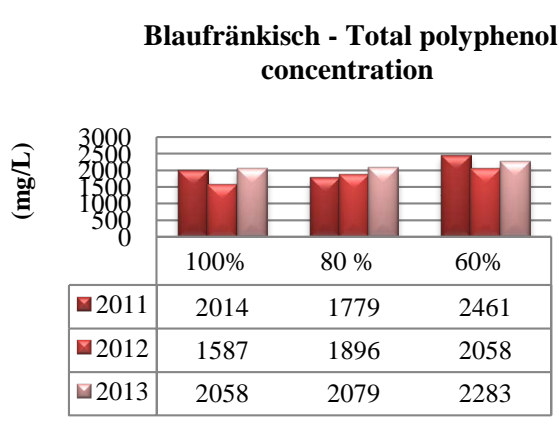

Figure 1. Total polyphenol content of Blafränkisch in 3 load levels in 2011, 2012, 2013 vintages.

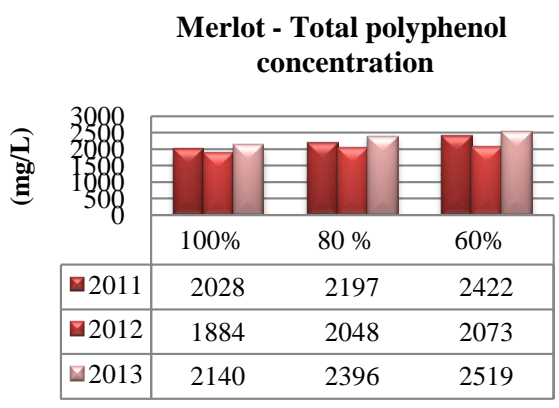

Figure 2. Total polyphenol content of Merlot in 3 load levels in 2011, 2012, 2013 vintages.

total polyphenol concentration in Blaufränkisch and Merlot wines at $60 \%$ load level managed to achieve the highest total polyphenol concentration in the produced wines. Organoleptic analyses were in accordance with the total polyphenol content of Blaufränkisch and Merlot wines. According to our experiments it can be summarized that the application of yield regulation leads to increased total polyphenol content. In the case of different load levels the amount of polyphenols did not show linearity, while the concentration of the appropriate phenolic components can be higher but even lower as it is shown later in our paper.

\subsection{Anthocyanins, Leucoanthocyanins and Catechins}

Anthocyanin, leucoanthocyanin, catechin contents of Blaufränkisch and Merlot wines are shown in Figure 3. According to the year of vintage and $60 \%-80 \%$ yield regulations different values were observed for the above mentioned polyphenols. The analysis of data proves the idea that besides vintage anthocyanin, leucoanthocyanin and catechin content of wines is in correlation with the variety of grape. In the case of the results of Merlot wine an increasing effect of yield regulation can be seen but in the case of Blaufränkisch rather stagnation can be observed, and leucoanthocyanin content in 2012 vintage was even decreased. Summarizing our results yield regulation did not lead to similar change of the different polyphenolic components (catechin, anthocyanin, leucoanthocyanin), besides increase of their concentration even concentration decrease was obtained. Winemakers can make decision according to our findings whether the amount of which phenolic component they intend to increase in the case of next vintage. They have to adjust the appropriate parameters in next year yield regulations to achieve the best quality wines.

\subsection{Color Intensity and Color Tone}

The measured and calculated color intensity and color tone values are presented in Figure 4. Results of the experiments represent that yield regulation does not have significant effect on color intensity and color tone of the produced red wine.

According to the results of our measurements increasing color intensity leads to decreasing color tone value. 

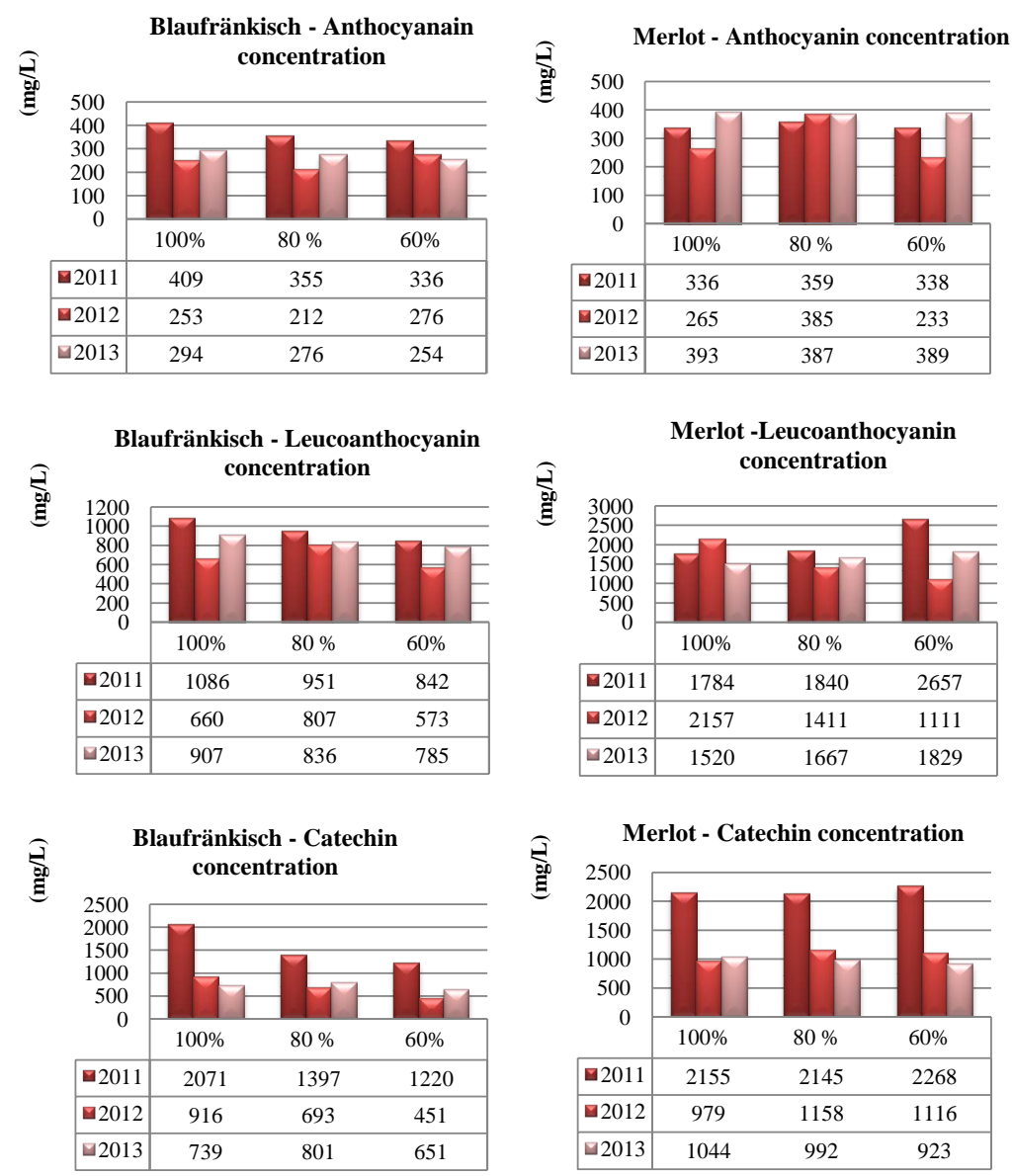

Figure 3. Anthocyanin, leucoanthocyanin and catechin content of Blafränkisch and Merlot wines in 3 load levels, in 2011, 2012, 2013 vintages.

Blaufränkisch - Color intensity value

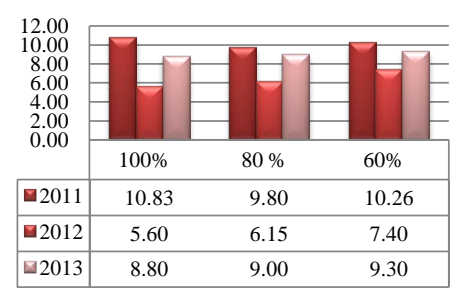

Blaufränkisch - Color tone value

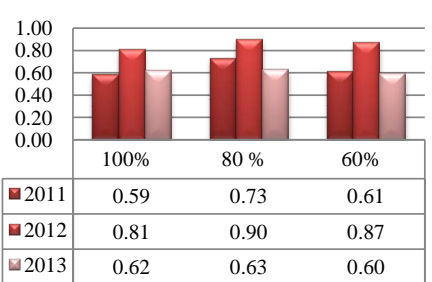

Merlot - Color intensity value

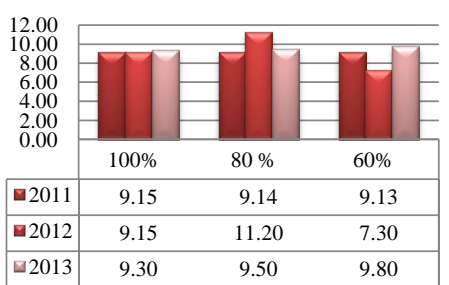

Merlot - Color tone value

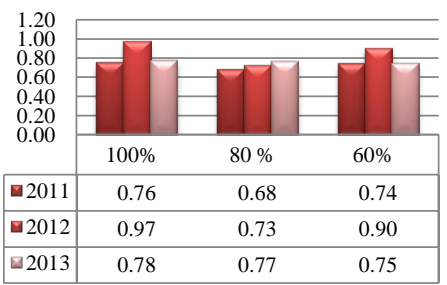

Figure 4. Color intensity and color tone of Blafränkisch and Merlot wines in 3 load levels, in 2011, 2012, 2013 vintages. Color intensity and color tone were measured on 520 and $420 \mathrm{~nm}$ by spectrometer. Color intensity: sum of the measured absorbance at the two wavelengths; color tone: quotient of the absorbance at the two wavelengths. 

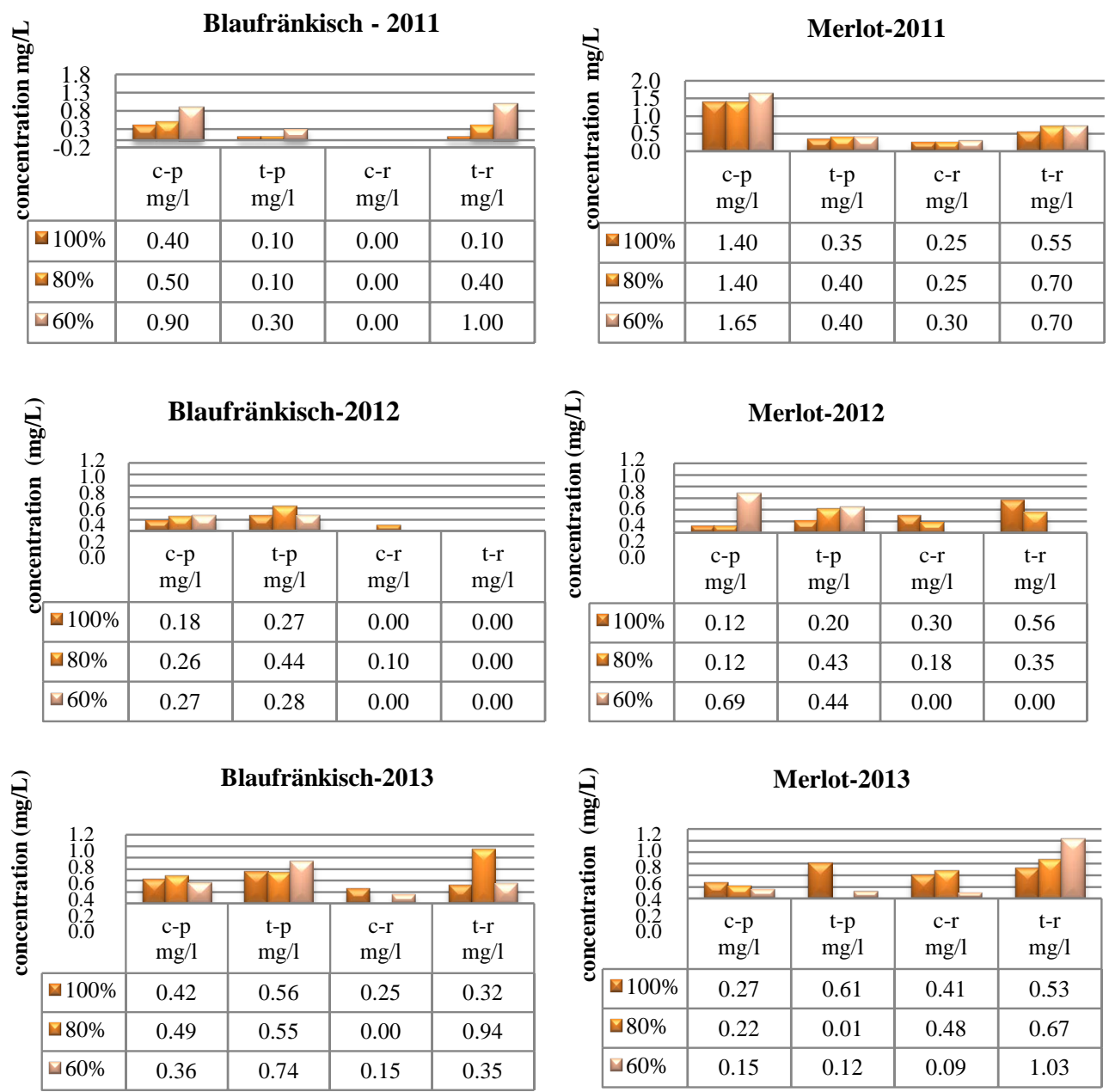

Figure 5. The effect of yield regulation on the amount of resveratrol and piceid of wines in vintages 2011, 2012, 2013; value of 0.00 means not detectable.(c-p: cis-piceid, t-p: trans-piceid, c-r: cis-resveratrol, t-r: trans-resveratrol).

Increasing value of color tone results in decreasing color intensity since color intensity and color tone values complement each other.

\subsection{Resveratrol and Piceid Content}

In the skin of grape only trans-piceid can be found which can be transformed to cis-piceid by UV irradiation. A small amount of cis-piceid may also appear in the skin of well ripen grape. Trans-resveratrol is a biologically active component of red grapes and wines, its biosynthesis performs from trans-piceid. Most of trans-resveratrol is formed in alcoholic fermentation from trans-piceid. Trans-resveratrol can be totally transformed to cis-resveratrol by UV treatment. During production of wines light should be avoided. It is known from literature that not only trans-piceid is the source of trans-resveratrol but there is another source in the berry that is viniferin. This is the reason that no equal material balance can be made between piceid and resveratrol.

Figure 5 contains the data of resveratrol and piceid concentrations of wines from our experiments. Based on the measurements on load levels of the three years it can be stated that trans-resveratrol content can be increased by applying yield regulation in the vineyard. The concentration of trans-piceid can also be increased by yield regulation, the highest concentration of trans-resveratrol and trans-piceid can be achieved at $60 \%$ load level. The concentration of resveratrol and piceid depends not only on yield regulation but also on the vintage. The effect of vintage cannot be forgotten, but in the case of the examined red wines it was not considerable. According to our previous results the effect of vintage on the quality of wines is more definite in white wines. 


\section{Conclusion}

In our experiments we managed to reveal the effect of yield regulation on the main wine parameters like polyphenol content and organoleptic properties. In the case of Blaufränkisch and Merlot the correlation between increased polyphenol content and wine quality was proven applying yield regulation in the vineyard. Based on our measurements it can be stated that total polyphenol content of wines increases by yield regulation. The effect of yield regulation on certain polyphenol components (anthocyanin, leucoanthocyanin, catechin) showed significant differences, besides increase and decrease in concentration may happen. Polyphenol components in wine samples made from grape of different yield regulation scales do not show linearity. In most cases $60 \%$ yield regulation results in higher concentrations in the case of certain components while sometimes the highest values are obtained in the case of $80 \%$ yield regulation. According to our results certain polyphenol components of wine can be designed by winemakers using appropriate yield regulation in the vineyard. The corresponding level of yield regulation determines the main wine parameters and polyphenol components. In the case of bad vintage yield regulation can be the way to the production of high quality wine. Besides examining yield regulation parameters location of the vineyard is also very important (mostly the number of sunny hours, temperature, amount of rain).

\section{Acknowledgements}

Authors thank the Hungarian Government for financial support of this work.

\section{References}

[1] Winkler, A.J., Cook, J.A., Kliewer, W.M. and Lider, L.A. (1974) General Viticulture. University of California Press, Berkeley.

[2] Bravdo, B., Hepner, Y., Loinger, C., Cohen, S. and Tabacman, H. (1984) Effect of Crop Level on Growth, Yield and Wine Quality of a High Yielding Carignane Vineyard. American Journal of Enology \& Viticulture, 35, $247-252$.

[3] Smart, R. and Robinson, M. (1991) Sunlight into Wine: A Handbook for Wine Grape Canopy Management. Winetitles, Underdale.

[4] Bravdo, B., Hepner, Y., Loinger, C., Cohen, S. and Tabacman, H. (1985) Effect of Crop Level and Crop Load on Growth, Yield, Must and Wine Composition, and Quality of Cabernet Sauvignon. American Journal of Enology \& Viticulture, 36, 125-131.

[5] Murisier, F. and Zufferey, V. (1996) Optimization of Shoot Load of Grapevine: Trial with Chasselas. Revue Suisse de Viticulture, Arboriculture, Horticulture, 28, 131-137.

[6] Carbonneau, A. (1997) General Relationship within the Whole-Plant: Examples of the Influence of Vigour Status, Crop Load and Canopy Exposure on the Sink "Berry Maturation" for the Grapevine. Acta Horticulturae, 427, 99-118.

[7] Monagas, M., Bartolome, B. and Gomez-Cordoves, C. (2005) Update Knowledge about the Presence of Phenolic Compounds in Wine. Critical Reviews in Food Science and Nutrition, 45, 85-118. http://dx.doi.org/10.1080/10408690490911710

[8] Gusman, J., Malonne, H. and Atassi, G. (2001) A Repraisal of the Potential Chemopreventive and Chemotherapeutic Properties of Resveratrol. Carcinogenesis, 22, 1111-1117. http://dx.doi.org/10.1093/carcin/22.8.1111

[9] Esparza, I., Santamaria, C., Calvo, M.I. and Fernández, J.M. (2009) Composition and Analysis of Colloidal Matter along Wine-Making, Exploitation of Its Antioxidant Activity in Final Stabilization Residues. Microchemical Journal, 91, 32-39. http://dx.doi.org/10.1016/j.microc.2008.07.002

[10] Boulton, R. (2001) The Copigmentation of Anthocyanins and Its Role in the Color of Red Wine: A Critical Review. American Journal of Enology and Viticulture, 52, 67-87.

[11] Auger, C., Al-Awwadi, N., Bornet, A., Rouanet, J.M., Gasc, F., Cros, G. and Teissedre, P.L. (2004) Catechins and Procyanidins in Mediterranean Diets. Food Research International, 37, $233-245$. http://dx.doi.org/10.1016/j.foodres.2003.11.008

[12] Gurbuz, O., Goçmen, D., Dagdelen, F., Gursoy, M., Aydin, S., Sahin, I., Buyukuysal, L. and Usta, M. (2007) Determination of Flavan-3-ols and Trans-Resveratrol in Grapes and Wine Using HPLC with Fluorescence Detection. Food Chemistry, 100, 518-525. http://dx.doi.org/10.1016/j.foodchem.2005.10.008

[13] Šeruga, M., Novak, I. and Jakobek, L. (2011) Determination of Polyphenols Content and Antioxidant Activity of Some Red Wines by Differential Pulse Voltammetry, HPLC and Spectrophotometric Methods. Food Chemistry, 124, 1208-1216. http://dx.doi.org/10.1016/j.foodchem.2010.07.047 
[14] Lorrain, B., Ky, I., Pechamat, L. and Teissedre, P.L. (2013) Evolution of Analysis of Polyhenols from Grapes, Wines, and Extracts. Molecules, 18, 1076-1100. http://dx.doi.org/10.3390/molecules18011076

[15] Mark, L., Pour Nikfardjam, M.S., Avar, P. and Ohmacht, R. (2005) A Validated HPLC Method for the Quantitative Analysis of Trans-Resveratrol and Trans-Piceid in Hungarian Wines. Journal of Chromatographic Science, 43, 445449. http://dx.doi.org/10.1093/chromsci/43.9.445

[16] Ratola, N., Luís-Faria, J. and Alves, A. (2004) T-Resveratrol in Wines from Alentejo. Food Technology and Biotechnology, 42, 125-130.

[17] Balga, I., Kiss, A., Gál, L., Leskó, A. and Kállay, M. (2014) Evaluating the Correlation between Chemical and Sensory Compounds in Blaufränkisch and Cabernet Franc Wines. Wine Studies, 3, 4469. http://dx.doi.org/10.4081/ws.2014.4469

[18] Slinkard, K. and Singleton, V.L. (1977) Total Phenol Analysis: Automation and Comparison with Manual Methods. American Journal of Enology and Viticulture, 28, 49-55.

[19] Ribéreau-Gayon, P. and Stonestreet, E. (1966) Le dosage des tannins du vin rouge et la détermination de leur structure. Chimie Analitique, 48, 188-196.

[20] Flanzy, M., Aubert, S. and Marinos, M. (1969) New Technique for Determination of Leucoanthocyanic Tannins. Annales de Technologie Agricole, 18, 327-328.

[21] Rebelein, H. (1969) Beitrag zur Bestimmung des Catechingehaltes in Wein. Deutsche Lebensmittel Rundschau, 61, 182-183. 